\title{
PHOTOGRAPHIC WIDE-FIELD IMAGING FROM SCHMIDT PLATES - I
}

\author{
H.H. HEYER, J. QUEBATTE and H. ZODET \\ European Southern Observatory \\ Garching \\ Germany
}

\section{Introduction}

The recent years have seen a clear trend in observational astronomy towards digital detectors, but they are only able to cover sky fields which are significantly smaller than what is possible with photographic plates. In consequence, there has been a tendency to concentrate on small sky areas and individual objects. Nevertheless, many large-scale structures can only be well comprehended if the observed fields are much larger than such CCD-frames. Similarly, the use of more than one passband adds important information for a better understanding of the nature of large structures. We demonstrate this by showing here a two-field composite of IC 1396 (Palomar/ESO Atlas), and a four-field composite from the ESO R-Atlas, covering an area of more than 100 square degrees around IC 4628 in Scorpius/Ara.

\section{Technical Information}

Two-colour, two-field composite of IC 1396. The plates 146-R and 188-B were directly amplified on a diffuse light contact printer onto Agfa RA $711 \mathrm{p}$ film and developed in an Ilford 2150 positive developing machine. The resulting films are best suited for copying on B/W papers of grade 3-5. As the Ilfochrome colour paper is much softer compared to the B/W papers, the contrast had to be raised by copying onto Kodak LPD 7 direct positive film, again developed in the Ilford machine. While making the colour composite, a register system was used for paper superposition. Each field was exposed through Kodak Wratten Tricolour filters blue and red on a Durst Condenser enlarger onto Ilfochrome Classic CPS 1.K paper.

Four-field B/W composite of area around IC 4628. The image was produced from four directly enhanced ESO $1 \mathrm{~m}$ Schmidt plates. After contact printing onto B/W paper, the composite was made in the traditional way by accurate cutting and mounting. The main technical problem was to produce mutually consistent copies of the 4 fields, despite differences in the sensitometric properties of the original plates. 


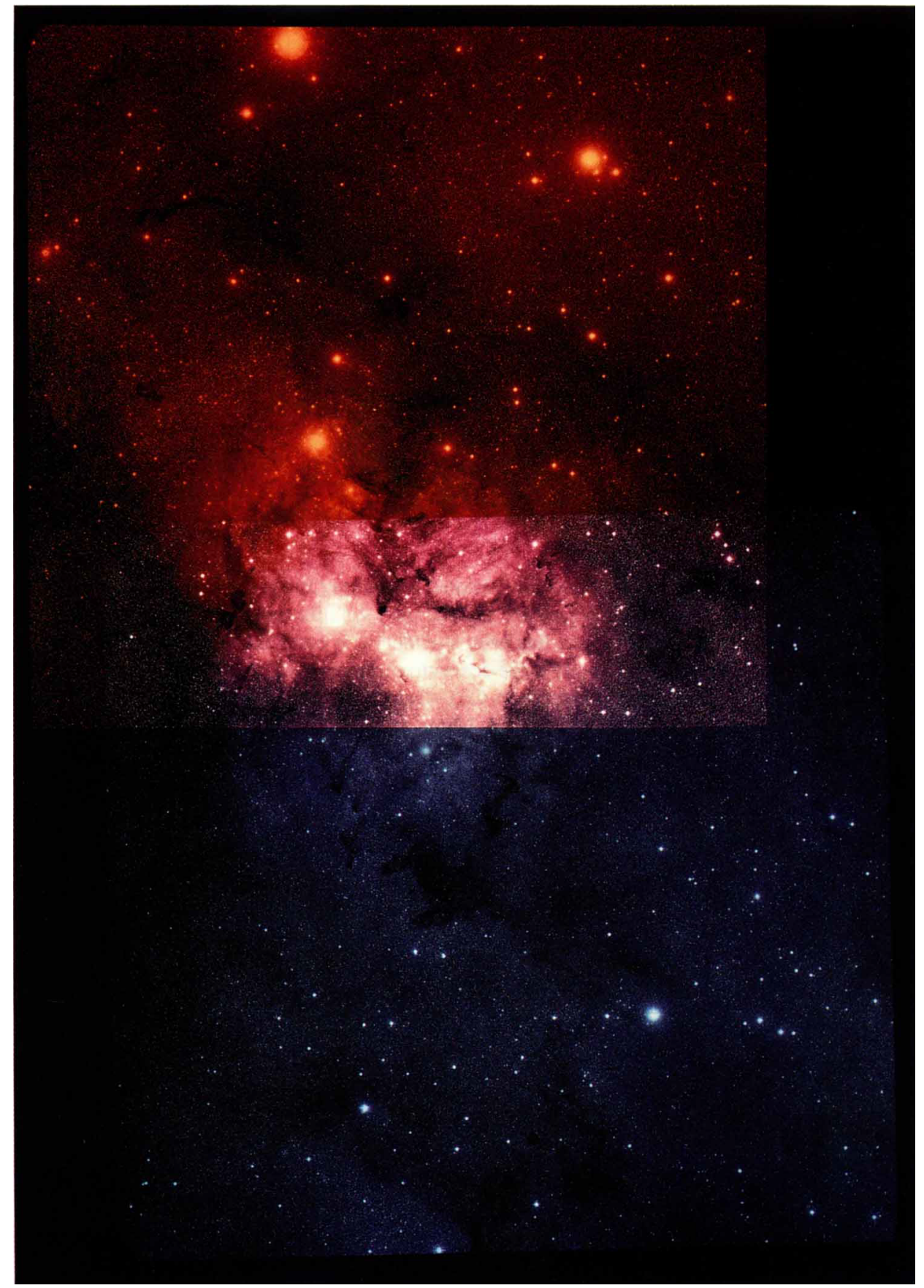

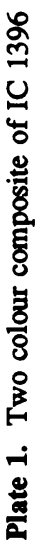




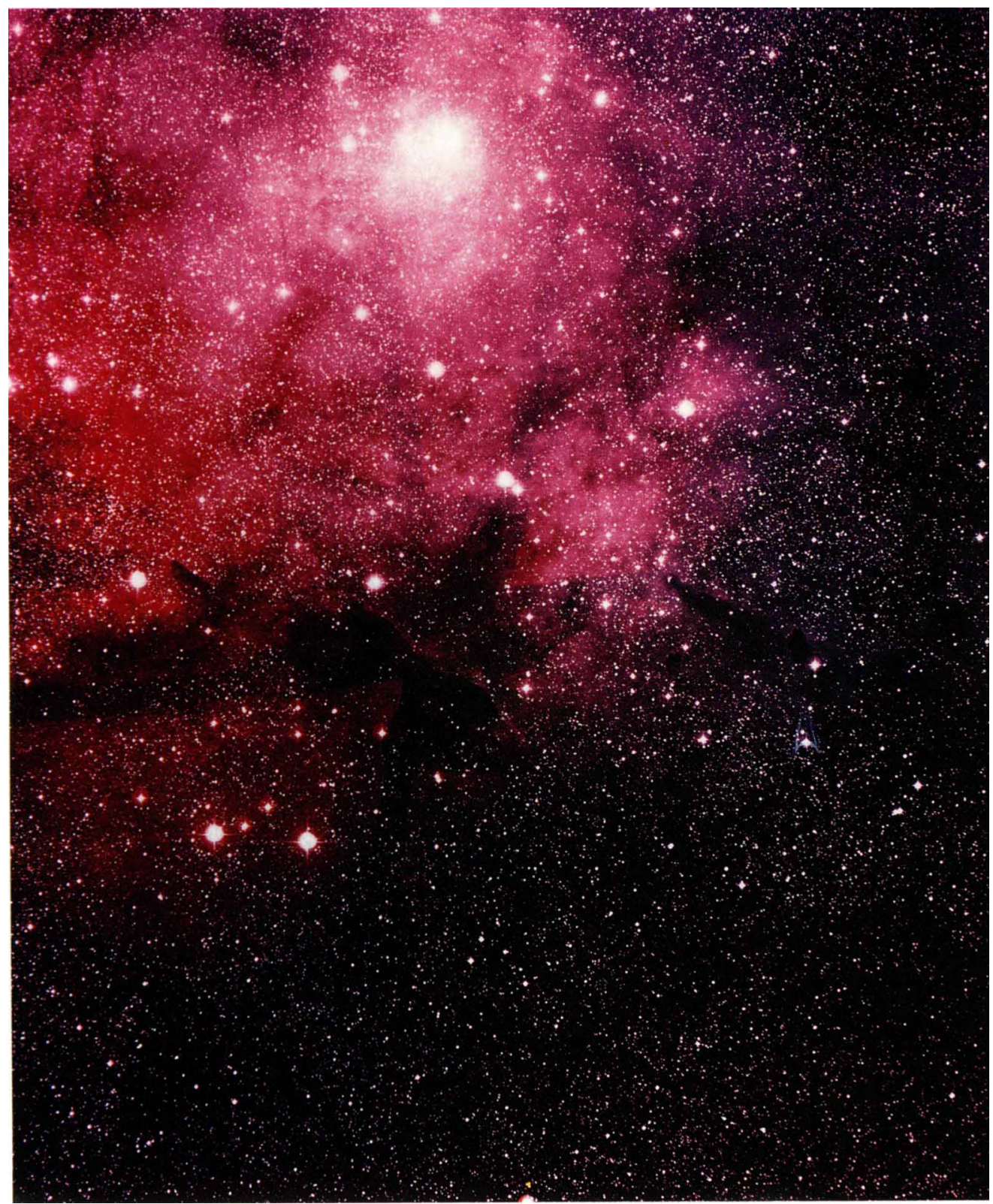

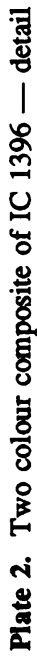




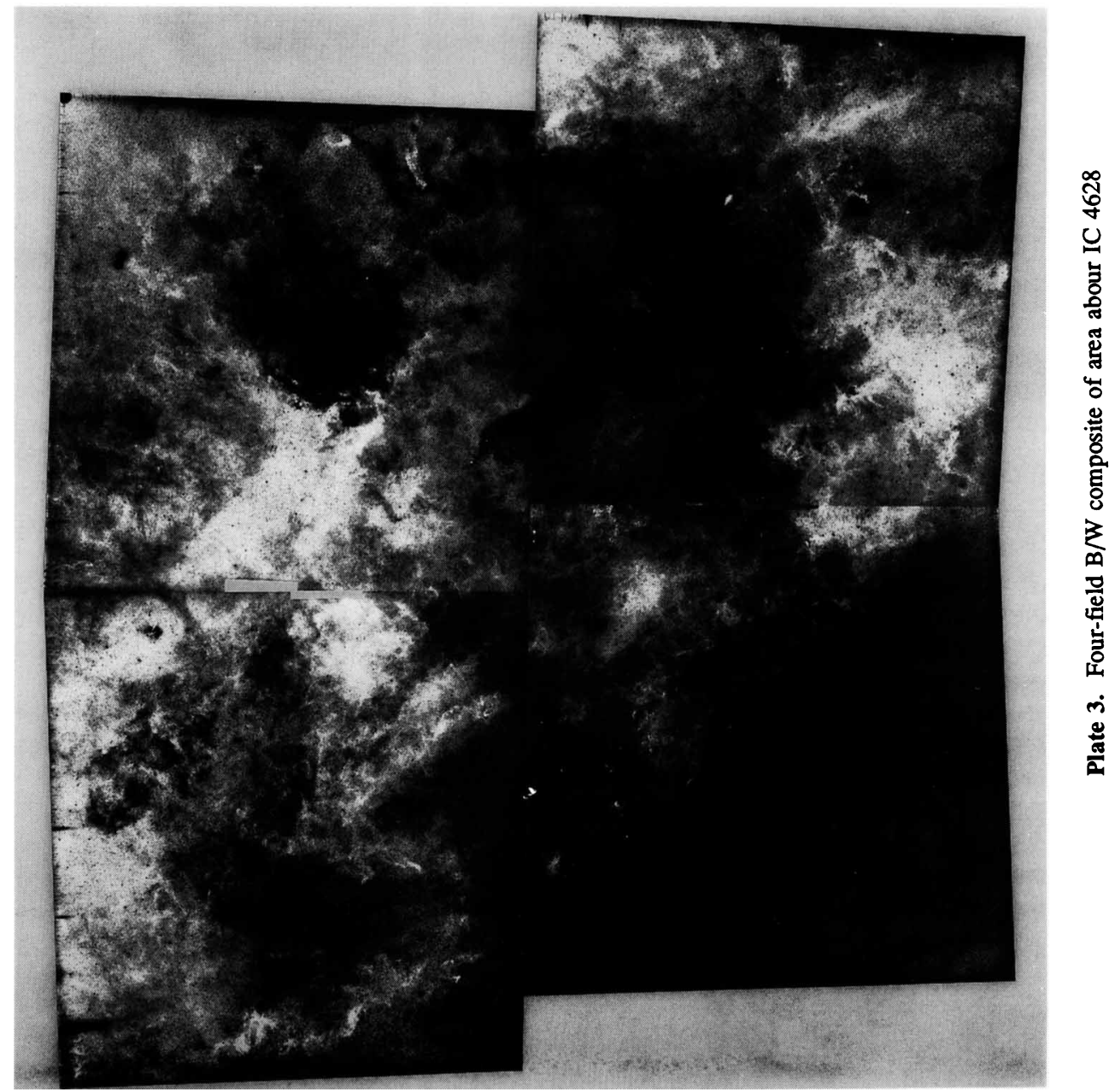




\section{Scientific Advantages}

The composite of the area around IC 1396 covers a field of $6^{\circ} \times 10^{\circ}$ and shows the large extent of the nebula. The overlapping region illustrates the advantage of colour information, both in the nebula and for individual stars. Note, however, that some of the rectilinear shapes in the nebula, shown in the detail print, are at least partly due to the reflection pattern of a bright star seen in the upper right of the red plate. The four-field $\mathrm{B} / \mathrm{W}$ composite shows a region of $10^{\circ} \times 10^{\circ}$ at the border between Scorpius and Ara. It contains a bewildering complex of stars, dark and bright nebulae of different shapes, stretching over several degrees. The combination of large area and high resolution not only provides an excellent overview of the field, it also facilitates the selection of specific areas for further studies with narrow-field instruments.

\section{Acknowledgement}

We would like to thank Dr. R. West for helping us to realize the poster as well as Dr. D. Malin for fruitful remarks on the finished result. 